\title{
Assessment of adipogenic, antioxidant, and anti-inflammatory properties of whole and whey bovine colostrum
}

\author{
Aejin Lee, ${ }^{1}$ Mariana C. F. Pontin, ${ }^{2}$ Erica Kosmerl, ${ }^{3}$ Rafael Jimenez-Flores, ${ }^{3}$ Débora B. Moretti, ${ }^{2 *}$ \\ and Ouliana Ziouzenkova ${ }^{1 *} \dagger$ \\ ${ }^{1}$ Department of Human Nutrition, The Ohio State University, Campbell Hall, 1787 Neil Ave., Columbus 43210 \\ ${ }^{2}$ Department of Animal Science, Luiz de Queiroz College of Agriculture, University of São Paulo, Av. Padua Dias, 11, Piracicaba, SP, \\ Brazil 13418-900 \\ ${ }^{3}$ Department of Food Science and Technology, The Ohio State University, Parker Food Science and Technology, 2015 Fyffe Road, \\ Columbus 43210
}

\section{ABSTRACT}

Bovine colostrum (BC) has been used for nutraceutical purposes for animals and humans. Bovine colostrum is a complex heterogeneous product and its antimicrobial activity, antioxidant potential, and growth factors can vary depending on age and species of the cow as well as their environment. Bovine colostrum preparation in skimmed or whey fractions can also alter properties of BC. Our goal was to compare cumulative anti-inflammatory, antioxidant, and adipogenic properties of natural (whole) versus whey BC. We compared properties of whole and whey BC in 3T3-L1 preadipocytes permanently transfected with reporters responding to changes in inflammatory $(\mathrm{N} f \kappa b \mathrm{RE} / \mathrm{green}$ fluorescent protein), anti-inflammatory (Nrf2/YFP), and adipogenic (Fabp 4/cyan fluorescent protein) status in cells. Interleukin- 6 secretion in these cells was measured by ELISA. Whole and whey BC induce IL-6 secretion from 3T3-L1 fibroblasts; however, whey preparation stimulated less IL-6 secretion. Cumulative inflammatory nuclear factor $(\mathrm{NF}) \kappa \mathrm{B}$ activation in the presence of lipopolysaccharide was reduced by both whole $(-27 \%)$ and whey BC $(-22 \%)$ compared with lipopolysaccharide-treated cells $(100 \%)$. Treatment with whole $\mathrm{BC}$ was more effective in the reduction of $\mathrm{NF} \kappa \mathrm{B}$ activation compared with whey $\mathrm{BC}$ and occurred in a dose-dependent manner. In consonance with decreased NFkB activation, the Nrf2 promoter activity was also reduced in response to whole $(-27 \%)$ and whey $(-13 \%)$ treatments compared with nontreated cells $(100 \%)$. Whole and whey BC suppressed adipogenesis, measured as induction of Fabp 4 , by -27 and $-13 \%$, respectively, compared with nontreated 3T3-L1 fibro-

Received February 18, 2019.

Accepted May 29, 2019.

*Both laboratories contributed equally to this work.

†Corresponding author: Ziouzenkova.1@osu.edu blasts (100\%). Our results showed distinct differences in properties of whey and whole BC that could be used to attain reduced adipogenic or cumulative inflammatory responses.

Key words: inflammation, adipogenesis, antioxidant, lacteal secretion, cytokine

\section{INTRODUCTION}

There is growing interest in human consumption of bovine colostrum (BC), the first mammary lacteal secretion, due to its nutritional properties as well as for pharmaceutical purposes (Ahmadi et al., 2011). Colostrum in its whole form consists of a casein fraction and a whey fraction, which has a high presence of biologically active molecules, among antibodies (Korhonen and Pihlanto, 2007). In addition to these macromolecules, a source of passive immunity for newborn ruminants, whey colostrum has enzymes with antioxidant and antimicrobial activity, minerals, hormones, and growth factors (Boudry and Thewis, 2009; Pandey et al., 2011). These and other components, including lactoferrin and vitamins $\mathrm{A}, \mathrm{C}$, and $\mathrm{E}$, contribute to antioxidant activity of whole colostrum. The mechanism of action of lactoferrin, for example, includes binding of iron, which is a powerful oxidant, and oxidative stress plays a major role in inflammation-induced diseases (Yadav et al., 2016). Other biological molecules, such as lactoperoxidase, lysozyme, cytokines, and oligosaccharides, also confer antimicrobial and anti-inflammatory properties in colostrum (Yadav et al., 2016).

An et al. (2009) reported anti-inflammatory effects of $\mathrm{BC}$ in intestinal epithelial cells stimulated by IL-1 $\beta$ (An et al., 2009). The authors observed BC-mediated suppression of the nuclear factor $(\mathbf{N F}) \kappa \mathrm{B}$ transcription factor activation via different mechanisms, including inhibition of the inhibitor protein of $\mathrm{NF \kappa B} \alpha$ degradation, and blocked translocation of p65 into the nucleus. This inhibition of $\mathrm{NF} \kappa \mathrm{B}$ resulted in the suppression 
of cyclooxygenase- 2 protein, as well as IL-8 and intracelullar adhesion molecule-1 expression levels. This study suggests that BC may have therapeutic potential against intestinal inflammation suppressing inflammatory cytokines. On the other hand, colostrum contains inflammatory cytokines including IL-6, IL-8, IL-10, and tumor necrosis factor $\alpha$. The concentrations of these cytokines in colostrum can be increased in pathological settings enhancing the proinflammatory potential of colostrum (Zanardo et al., 2007). Therefore, it is critical to assess the cumulative inflammatory properties of colostrum given that age, species, and environment of the cows contribute to variation in colostrum composition. All biologically active proteins (except casein) are associated with the whey fraction and are dependent on the multiple variables described above, as well as the time of lactation and differences between primiparous and multiparous cows (Raimondo et al., 2019). Whey colostrum preparation compared with whole colostrum influences concentrations of lipophilic antioxidants, SFA, PUFA, cytokines, and antioxidant proteins. Collectively these changes in the antioxidant potential can influence anti-inflammatory properties in whey compared with whole colostrum. The effects of whole or whey formulations on inflammation and antioxidant properties have not been compared.

Nuclear factor (erythroid-derived 2)-like 2 (NRF2) and $\mathrm{NF \kappa B}$ are the 2 master regulators of cellular responses to oxidative stress and inflammation, respectively (Wardyn et al., 2015). To assess the cumulative effect on inflammation in response to nutrients with a complex composition, preadipocyte fibroblast reporter cell lines containing $\mathrm{NF} \kappa \mathrm{B}$ response element ( $\mathrm{N} f \kappa b$-RE) have been used (Baker et al., 2011). Translocation of p65/p50 complex to the nucleus activate $\mathrm{N} f \kappa b$-RE (Wardyn et al., 2015). In a similar fashion, activation of Nrf2-RE was used to assess antioxidant response of nutrients (Shen et al., 2014). The exposure to lipid-rich nutrients, such as colostrum, could have a long-term effect on metabolism via regulation of adipogenesis, development of adipose tissue, chronic inflammation related to obesity, or a combination of these (Yamashita et al., 2018). Adipogenesis is regulated by the nuclear factor peroxisome proliferator activated receptor gamma (PPAR $\gamma$; Rosen and Spiegelman, 2001). The activation of PPAR $\gamma$ leads to activation of its target gene, fatty acid binding protein 4 (Fabp 4 ; Tontonoz and Spiegelman, 2008). Activation of transcription factor PPAR $\gamma$ can also have anti-inflammatory effects due to its binding affinity to $\mathrm{p} 65$, preventing the activation of $\mathrm{N} f \kappa b$-RE during inflammation (Chen et al., 2003). To elucidate nutrient-dependent effects, Shen et al. (2014) developed biosensor preadipocyte cell lines $\mathrm{N} f \kappa b$-RE/ GFP (GFP = green fluorescent protein), Nrf2-P/
YFP (YFP = yellow fluorescent protein), and Fabp $4 /$ CFP $(\mathbf{C F P}=$ cyan fluorescent protein) for combined screening of the interrelated anti-inflammatory, antioxidant, and anti-adipogenic pathways. We hypothesize that differences in the composition of whole and whey $\mathrm{BC}$ will influence their anti-inflammatory, antioxidant, and anti-adipogenic properties. Our objective was to validate a high-throughput screening approach for comparison of whole and whey BC properties.

\section{MATERIALS AND METHODS}

\section{Preparation of Culture Medium and Differentiation Medium}

We purchased Dulbecco's modified Eagle medium (DMEM) from Gibco Life Technologies (\#11965, Grand Island, NY); high glucose with L-glutamine, without sodium pyruvate, penicillin/streptomycin (P/S) from Invitrogen (\#15140-122, Karlsruhe, Germany); new born calf serum (NBCS) from Gibco Life Technologies (\#16170-078); fetal bovine serum (FBS) from Gibco Life Technologies (\#10437-028); bovine insulin from Sigma-Aldrich (\#I-5500, St. Louis, MO); isobutylmethyxanthine (IBMX) from Sigma-Aldrich (\#I7018); dexamethasone from Sigma-Aldrich (\#D-4902); and dimethyl sulfoxide (DMSO) from ISC-Bioexpress (\#0231, Kaysville, UT). Lipopolysaccharide (LPS) was purchased from Sigma-Aldrich (\#L2762), retinoic acid from Sigma-Aldrich (\#R2625), and rosiglitazone from Enzo Life Sciences (\#ALX-350-125-M100, Ann Arbor, MI).

The culture medium contained $1 \% \mathrm{P} / \mathrm{S}$ and $10 \%$ NBCS in DMEM. Two differentiation media were used. The first is termed differentiation medium I [DMEM containing $1 \% \mathrm{P} / \mathrm{S}$ and $10 \% \mathrm{FBS}$, bovine insulin $(1: 1,000)$, IBMX diluted in DMSO $(1: 1,000)$, and dexamethasone diluted in ethanol $(1: 10,000)]$. Differentiation medium II was prepared with the same reagents as differentiation medium I; however, medium lacked the IBMX and dexamethasone. Differentiation was induced in confluent preadipocytes by differentiation medium I (d 0). Differentiation medium II was added on d 2 and replaced every $48 \mathrm{~h}$.

\section{Collection and Processing of BC}

We compared whole and whey $\mathrm{BC}$ properties from 3 individual multiparous (second to fourth lactation) Jersey cows (age $3.8 \pm 0.5 \mathrm{yr}$ ). Samples were collected from the first lactation and whey colostrum extraction was performed within the first $24 \mathrm{~h}$ after collection. Samples in their natural form (whole colostrum) and extracted form (whey colostrum) were stored and ana- 
lyzed within 1 wk (with storage at $4^{\circ} \mathrm{C}$ ). To prepare whey colostrum, a sample of the lacteal secretion was centrifuged at $3,600 \times g$ at $4^{\circ} \mathrm{C}$ for 22 min to remove the lipid fraction. Whey colostrum was obtained after incubation with rennet (Chy-Max M from Chr Hansen, Hoersholm, Denmark, material \#127223, diluted as vol:vol $=1: 1,000$ to the sample) in a water bath for $30 \mathrm{~min}$ at $37^{\circ} \mathrm{C}$. After centrifugation at $16,639 \times \mathrm{g}$ at $4^{\circ}$ for $20 \mathrm{~min}$, the supernatant (whey colostrum) was collected and stored at $4^{\circ} \mathrm{C}$. The rennet control and whey colostrum contained the same concentrations of rennet (vol:vol $=1: 1,000$ rennet/PBS) in all cell-based experiments.

\section{T3-L1 Preadipocyte Cell Lines and Treatments}

3T3-L1 Expressing Nf^b-RE/GFP. The 3T3-L1 preadipocytes were previously stably transfected with the reporter $N f \kappa b$-RE/GFP (Shen et al., 2014). Cells were plated into 96 -well plate at $70 \%$ confluence in growth medium. After $24 \mathrm{~h}$ (d 0), the medium was replaced with differentiation medium I without FBS and following treatments: (1) nontreated control, (2) LPS (20 ng/mL in water), (3) $0.1 \%$ whole or whey BC, and (4) $0.5 \%$ whole or whey BC. On d 2, differentiation medium I was replaced with differentiation medium II containing the following treatments: (1) nontreated control, (2) LPS (20 ng/mL), and (3 and 4) 0.1 and $0.5 \%$ whole and whey BC with LPS $(20 \mathrm{ng} / \mathrm{mL})$. Twentyfour hours afterward, upon harvest, the differentiation medium was collected and analyzed for secreted IL-6. The GFP was measured in the living cells at $485 / 528$ $\mathrm{nm}$ wavelengths for excitation and emission (Ex/Em). Protein content was measured in the washed and lysed cells.

3T3-L1 Preadipocytes Expressing Nrf2/YFP. The 3T3-L1 preadipocytes previously transfected with the reporter Nrf2/YFP (Shen et al., 2014) were used. Cells were handled as in 3.3.1. Nrf2/YFP cell activity was validated by comparison of cells stimulated with ethanol (vehicle, 0.1\%) or retinoic acid (RA, $100 \mathrm{n} M$ in $0.1 \%$ ethanol), a canonic regulator of this pathway. After $24 \mathrm{~h}$ (d 0), the medium was replaced with differentiation medium I without FBS and Nrf2/YFP cells were stimulated with 0.1 and $0.5 \%$ whole $\mathrm{BC}$, whey $\mathrm{BC}$, or remained untreated (control). On the $\mathrm{d} 2$, the differentiation medium I was replaced with the differentiation medium II containing the same treatment used on d 0. Twenty-four hours afterward, the YFP in the living cells was determined at 500/530 nm Ex/Em. Protein was measured in the washed and lysed cells.

3T3-L1 Preadipocytes Expressing Fabp//CFP. The 3T3-L1 preadipocytes previously transfected with the reporter Fabp4/CFP were used (Shen et al., 2014).
Cells were handled as in the previous section. Fabp $4 /$ CFP cell activity was validated by comparison of cells stimulated with ethanol (vehicle, $0.1 \%$ ) or rosiglitazone (1 $\mu M$, BRL-49653), an agonist of this pathway. After $24 \mathrm{~h}(\mathrm{~d} 0)$, the medium was replaced with differentiation medium I without FBS containing 0.1 and $0.5 \%$ whole or whey BC, or were not treated (control). On d 2 , the differentiation medium I was replaced with differentiation medium II containing the same treatments. The CFP in the living cells was determined at 426/460 $\mathrm{nm}(\mathrm{Ex} / \mathrm{Em}) 48 \mathrm{~h}$ after treatment. Protein content was measured in the washed and lysed cells.

\section{IL-6 and Protein Determination}

Interleukin-6 in culture medium and whole and whey BC samples were measured by an ELISA kit (Invitrogen, Grand Island, NY). The protein content in RIPA cell lysates was measured using a BCA kit (Thermo Fisher Scientific, Rockford, IL).

\section{Statistical Analysis}

Data represents measurements using whey and whole colostrum from 3 cows. Whey and whole $\mathrm{BC}$ from each cow is presented as an average of 8 measurements performed in independent settings. Data are shown as mean \pm standard error of the mean. Group comparisons were performed using paired or unpaired Student's $t$-test, considering a probability of $5 \%(P<0.05)$. The IBM SPSS Statistics 23 software (IBM Corp., Armonk, NY) was used for analysis.

\section{RESULTS}

\section{Whole and Whey BC Fractions Inhibit LPS-Induced NFKB Activation in Adipocytes}

The 3T3-L1 fibroblasts are canonic experimental systems that allow for assessment of the effects of complex natural products on inflammation (Shen et al., 2015). Given that NFKB is a master regulator of inflammation, we used 3T3-L1 fibroblasts permanently transfected with $N f \kappa b$ response element $(N f \kappa b R E)$-GFP and activated by LPS (Figure 1). Activation of inflammation was measured as an increase in GFP in living cells that was normalized by protein content to account for cell proliferation. We also measured the concentrations of secreted IL- 6 that serve as a specific marker of inflammation in response to BC. Both whole and whey BC markedly increased IL-6 concentration to 340 and $181 \%$, respectively, compared with control LPSstimulated cells (100\%; Figure 1A). Both whole and whey BC exhibited dose-dependent stimulations of IL-6 
secretion. This increase is consistent with the presence of IL-6 in colostrum (Figure 1A, insert; Zanardo et al., 2007) and with the known multifunctional roles of IL-6 that plays both anti- and pro-inflammatory roles in different settings (Scheller et al., 2011). However, whole $\mathrm{BC}$ induced higher levels of IL-6 secretion from cells than whey $\mathrm{BC}$, even though whey colostrum contained higher endogenous levels of IL-6 (Figure 1A, insert). To elucidate cumulative inflammatory response, we measured the $N f \kappa b R E$ activation in the same adipocytes.

Stimulation of 3T3-L1 $(N f \kappa b R E)$-GFP fibroblasts with canonic inducer of inflammation LPS was not influenced by residual rennet from extraction (Figure 1B). Whole BC reduced LPS-dependent $N f \kappa b R E$ activation more effectively than whey $\mathrm{BC}(-27$ and $-22 \%$, respectively, compared with LPS-treated cells, $100 \%$; Figure 1C). Overall, the inhibition was more pronounced by whole $\mathrm{BC}$ than its whey fraction at the $0.5 \%$ concentration.

\section{Whole and Whey BC Alter Antioxidant Potential}

Inflammatory response depends on the redox status in the cells regulated by Nrf2 (Wardyn et al., 2015). We measured oxidative stress in 3T3-L1 adipocytes transfected with Nfr2-YFP reporter in response to whole and whey BC. The RA stimulation decreased Nfr2 activation in Nfr2-YFP-3T3-L1 adipocytes ( $-10 \%$ vs. vehicle-treated control), whereas rennet moderately increased Nfr2 activation at $0.1 \%$ concentration $(110 \%$ vs. nontreated control; Figure 2A). Treatment with whole $\mathrm{BC}$ led to moderate dose-dependent inhibition of Nfr2 activation to $73 \%$ compared with nonstimulated cells (100\%; Figure 2B). The whey BC reduced Nfr2 activation to a lesser extent than whole BC ( -0.3 vs. $-13 \%$ at $0.1 \%$ concentrations). Overall Nfr2 status in the cells correlated with the NFkB-dependent inflammatory response in cells stimulated with whole and whey BC (Figure 2C).

\section{Whole and Whey BC Reduce Adipogenic Responses 3T3-L1 Cells}

Adipose fatty acid binding protein $(F a b p 4)$ plays a critical role in fatty acid trafficking in preadipocytes and adipocytes that leads to activation of PPAR $\gamma$, a master regulator of adipogenesis. The 3T3-L1 preadipocytes were permanently transfected with Fabp 4-CFP reporter to assess the adipogenic potential of these preadipocytes. The Fabp4-CFP preadipocytes were significantly activated by PPAR $\gamma$ ligand BRL (124 vs. $100 \%$ vehicle, Figure 3A), consistent with the low expression of Pparg in 3T3-L1 preadipocytes. Rennet treatment led to significant but very low activation of

\section{A Inflammatory response in cells}
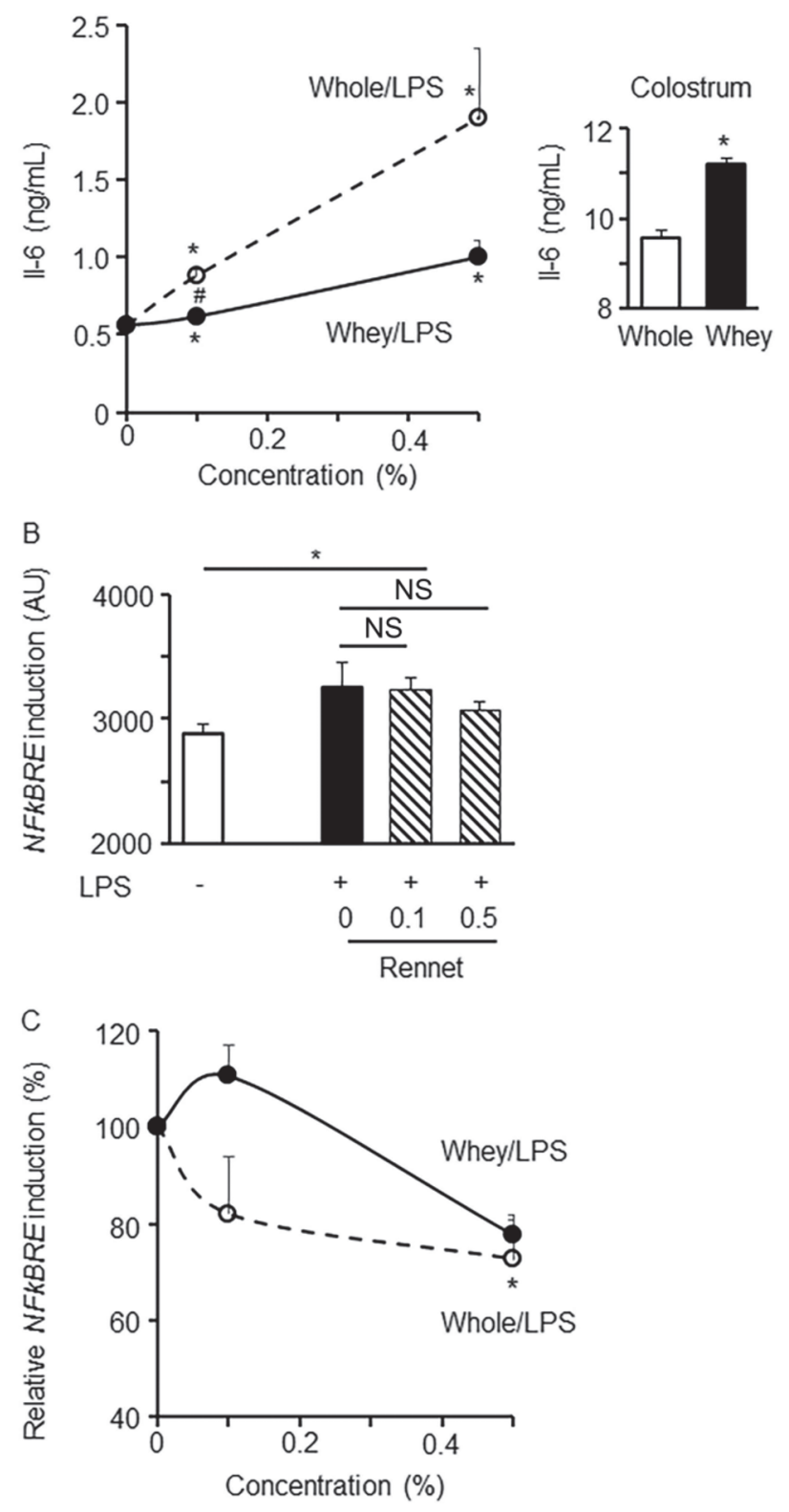

Figure 1. Whole and whey bovine colostrum (BC) increased IL-6

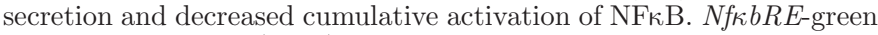
fluorescent protein (GFP) 3T3-L1 adipocytes were stimulated with LPS $(20 \mathrm{ng} / \mathrm{mL})$, rennet $(0.1$ or $0.5 \%)$, or indicated concentrations of whole (dashed line, open circles) and whey (solid line, solid circles) BC. Data represent measurements using whey and whole colostrum from 3 individual cows. Whey and whole BC from each cow is an average of 8 measurements performed in independent settings. Data are shown as mean \pm SEM. (A) IL-6 concentrations measured by ELISA in the culture medium collected $24 \mathrm{~h}$ after stimulation. ${ }^{*}$ Significance compared with LPS-stimulated control cells. \#Significance between whole versus whey samples $(P<0.05, t$-test). Insert shows IL-6 concentrations in the whole and whey $\mathrm{BC}$ samples that were used for stimulation. (B, C) Green fluorescent protein (GFP) measured in the same living $N f \kappa b R E$-GFP cells and normalized to protein concentrations. (B) Normalized GFP in 3T3-L1 adipocytes $24 \mathrm{~h}$ after stimulation with and without LPS and rennet. (C) Normalized GFP in 3T3-L1 adipocytes after LPS stimulation and treatment with whole or whey colostrum. Paired $t$-test. 

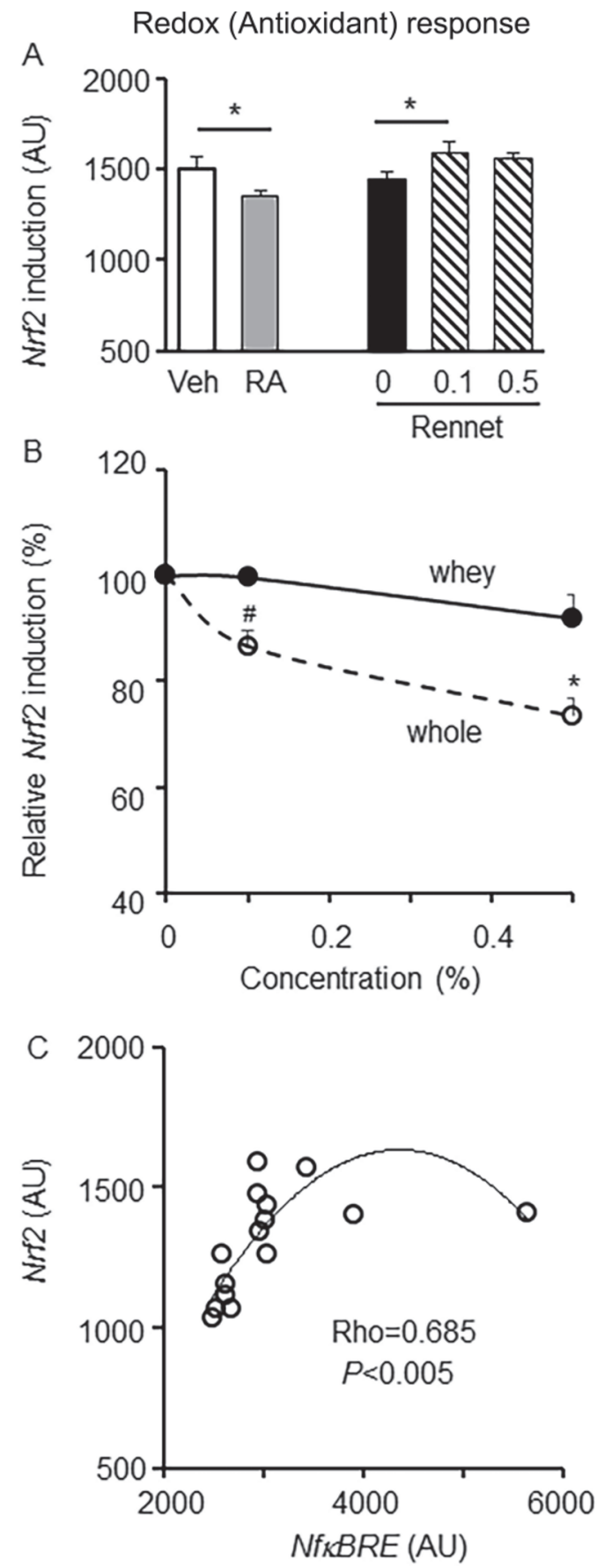

Figure 2. Whole and whey bovine colostrum (BC) inhibited activation of Nrf2 promoter. Data represent measurements using whey and whole colostrum from 3 individual cows. (A) Yellow fluorescent protein (YFP) measured in living Nfr2-YFP3T3-L1 adipocytes $24 \mathrm{~h}$ after stimulation with retinoic acid (RA, $100 \mathrm{n} M, \mathrm{n}=8$ ) and rennet $\left(0.1\right.$ or $0.5 \%, \mathrm{n}=8$; ${ }^{*}$ represents $P<0.05, t$-test). (B) Yellow fluorescent protein with same treatment as (A) as well as indicated concentrations of whole (dashed line, open circles) and whey (solid line, solid circles) BC from 3 different cows. Data were normalized to protein concentrations and are shown as mean \pm standard error of the mean. \#Significance between whole versus whey samples ( $t$-test). (C) Spearman correlation between Nfr2-YFP and NfאbRE-GFP 3T3-L1 adipocytes with and without activation by whole and whey BC. Veh $=$ vehicle; $\mathrm{AU}=$ arbitrary units.
Fabp 4 (105 and $106 \%$ activation vs. nontreated cells). Treatment with whole BC suppressed Fabp 4 activation dose-dependently, resulting in $-27 \%$ reduced activity (Figure 3B, vs. $100 \%$ in control cells). The effect of the protein-rich whey BC on Fabp 4 activation was less pronounced ( $-13 \%$ compared with control). Whole BC appeared to be more effective in suppression of adipogenic responses than the whey $\mathrm{BC}$ fraction.

\section{DISCUSSION}

Bovine colostrum has been extensively studied due to its large number of biologically active molecules (Boudry and Thewis, 2009; Pandey et al., 2011). Here we found that the anti-inflammatory, antioxidant, and anti-adipogenic properties are distinctly different between natural whole and whey BC fractions. Whole BC exerts stronger NFkB-dependent anti-inflammatory properties compared with whey fraction, which were related to the Nrf2-responsive oxidative status of these cells. However, whole BC also exhibited robust antiadipogenic properties. Our data suggest that whole and whey BC could have specific nutritional and biomedical applications based on the differences in the regulation of inflammatory and metabolic pathways that were assessed in high-throughput reporter assays.

Antioxidative potential of $\mathrm{BC}$ remains a subjects of debate (Yadav et al., 2016). In humans, increased cytokine levels in colostrum were associated with neonatal jaundice (Zanardo et al., 2007). Appukutty et al. (2012), in turn, observed that supplementation with $\mathrm{BC}$ protects against oxidative stress induced by exercise in the skeletal muscle tissue of mice (Appukutty et al., 2012). In our study, the overall effect of BC in living cells resulted in the inhibition of Nfr2 promoter activation. Thus, particularly whole BC, even in a small dose, could alter the redox status in cells. Several BC components could be responsible for the change in the redox status regulated by NRF2. Casein or lipid fractions (or both) of the whole BC may be involved in the regulation of antioxidant capacity. Casein is able to inhibit Fe-induced peroxidation of arachidonic acid inserted into multilamellar liposomes, indicating the antioxidant properties of milk and colostrum proteins (Cervato et al., 1999). Colostrum contains antioxidant enzymes, including catalase, glutathione peroxidase, and superoxide dismutase, as well as a high concentration (30 $\mathrm{mg} / \mathrm{L}$ ) of lactoperoxidase (Albera and Kankofer, 2009). Lactoperoxidase in the presence of hydrogen peroxide generates reactive species with high antimicrobial activity. Lactoferrin also provides antioxidant protection by binding free iron produced during cellular inflammation. Furthermore, colostrum's vitamins such as vitamins $\mathrm{A}, \mathrm{C}$, and $\mathrm{E}$ and minerals such as selenium, 


\section{Adipogenic response}
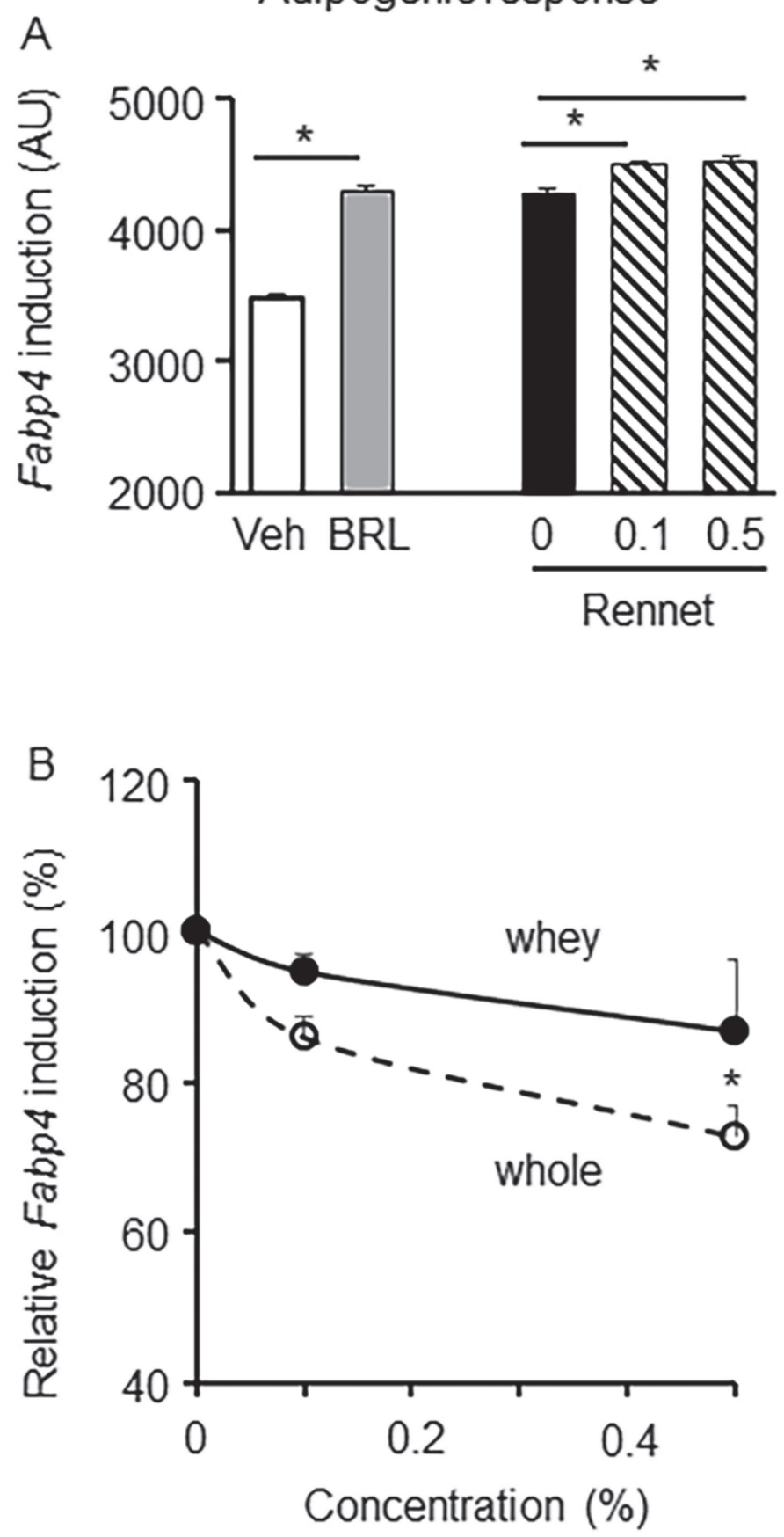

Figure 3. Whole and whey bovine colostrum (BC) inhibited activation of Fabp 4 promoter. Data represent measurements using whey and whole colostrum from 3 individual cows. (A) Cyan fluorescent protein (CFP) measured in living Fabp 4-CFP adipocytes $48 \mathrm{~h}$ after stimulation with rosiglitazone (BRL, $1 \mu M$ ) and rennet (0.1 or $0.5 \%$ ), ( $\mathrm{n}=8, t$-test). (B) Cyan fluorescent protein as treated in (A) as well as with indicated concentrations of whole (dashed line, open circles) and whey (solid line, solid circles) BC from 3 different cows. Data were normalized to protein concentrations and are shown as mean \pm SEM. $* P<0.05$ compared with control vehicle (Veh)-stimulated cells, $t$-test. $\mathrm{AU}=$ arbitrary units zinc, and manganese could act as antioxidants and/ or support antioxidative pathways, preventing the formation of reactive oxygen species (Ahmadi et al., 2011, Pandey et al., 2011). Glutathione in colostrum is often described as the ultimate antioxidant (Albera and Kankofer, 2009). Recent research has also focused on the assessment of overall effects of $\mathrm{BC}$ under oxidative stress, including neuroprotective effect of BC (Stewart, 2008; Choi et al., 2010; Janusz and Zablocka, 2010). Kim et al. (2012), in turn, reported that treatment with $\mathrm{BC}$ significantly decreased neuronal cell death induced by $N$-methyl-D-aspartic acid in the rodent hippocampus (Kim et al., 2012). Our study demonstrated that antioxidative effects of $\mathrm{BC}$ can be measured in vitro and the properties of whole $\mathrm{BC}$ were compared with its whey preparation. Glycomacropeptide, which is produced through cleavage of casein by rennet, is also reported to have an antioxidant function (Neelima et al., 2013). In our studies, whole colostrum had a more profound effect on Nrf2, indicating the higher antioxidant potential of whole compared with whey BC. Although glycomacropeptide may contribute to the effect of whey $\mathrm{BC}$, the overall antioxidative effects of whey $\mathrm{BC}$ were moderate in our study. The dual assessment of whole and whey BC in $N r f 2$ and $N f \kappa B$ reporter systems suggests that antioxidant properties of whole and whey $\mathrm{BC}$ were major determinants of the cellular inflammatory response. The Nfr2 promoter activity positively correlated with $N f \kappa B$ activation in cells stimulated by whole and whey $\mathrm{BC}$. Whole $\mathrm{BC}$ composition containing lipophilic antioxidants appeared to have marked antiinflammatory and antioxidant potentials that exceed those seen with whey BC.

Both whey and whole BC markedly reduced $N f \kappa b R E$ activation at $0.5 \%$ concentrations compared with control nonstimulated cells, in spite of having endogenous proinflammatory cytokines, such as IL-6. These data indicate that $\mathrm{BC}$ has a great potential to decrease the inflammatory signaling cascade. An et al. (2009) also observed that $\mathrm{BC}$ protects intestinal epithelial cells from inflammation by inhibiting the NFkB pathway (An et al., 2009). The authors observed that $\mathrm{BC}$ inhibited IL-13-induced IL-8, intracellular adhesion molecule-1 mRNA expression, IL-1 $\beta$-induced NFkB activation, cyclooxygenase- 2 protein expression, and inhibitor protein of $\mathrm{NF \kappa \textrm {B }}(\mathrm{I} \kappa \mathrm{B} \alpha)$ degradation, besides blocking translocation of p65 into the nucleus. Based on the studies

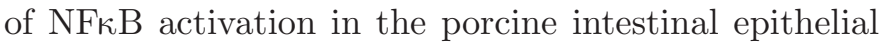
cell (IPEC-J2) stimulated by heat-inactivated bacteria Escherichia coli and Salmonellae enterica, Blais et al. (2015) proposed to use whey BC as a feed additive to prevent infections. In these studies, whey BC decreased the expression of early and late inflammatory genes 
(Blais et al., 2015). In our study, both whole and whey BC could suppress NF $\kappa$ B response with similar efficacy.

Several studies showed that IL-6 is a multifunctional cytokine underlying many physiologic processes in addition to its role in inflammation (Scheller et al., 2011). Although $N f \kappa b R E$ activation was suppressed, both whey and whole BC increased IL-6 secretion, suggesting that this cytokine plays a physiologic role in neonates. The secretion of IL-6 plays an important role in proliferation and repair of the intestine, where colostrum is absorbed (Kuhn et al., 2014). It is possible that the presence of IL-6 in the colostrum (Zanardo et al., 2007) and the stimulation of IL-6 secretion by BC could play a role in stimulating the development of the newborn intestinal epithelium (Garg et al., 2018). In the present work, our data suggest that measurement of $N f \kappa b R E$ activation provides improved assessment of inflammation in response to complex secretory nutrients, such as $\mathrm{BC}$, than specific cytokines such as IL-6.

The effects of $\mathrm{BC}$ on adipogenesis and the mechanisms involved in this regulation have been limited (Lyle et al., 1998; Lee and Hossner, 2002). Moreover, considering that $\mathrm{BC}$ is a complex heterogeneous product, it is inherently variable and can display different effects depending on environment, feed, age, and species of the cow. The BC formulations, such as natural, skimmed, or whey, can also alter adipogenic properties of BC. Enrichment of $\mathrm{BC}$ in proteins, fats, minerals, and vitamins, and the presence of molecules essential for promoting growth and development of the gastrointestinal tract of newborns all can influence adipogenesis (Garg et al., 2018). Growth factors, such as IGF and epidermal growth factor, are known to increase proliferation and differentiation of intestinal cells, influencing the absorption capacity of electrolytes and nutrients by newborns (Pandey et al., 2011). It is also known that IGF can stimulate a variety of cells, among them 3T3-L1 adipocytes (Boney et al., 2001). Plateletderived growth factor is also present in high amounts in colostrum and could induce mitosis of 3T3-L1 cells (Bachmeier and Loffler, 1995). Multiple studies (Shing and Klagsbrun, 1987; Belford et al., 1997; Lee and Hossner, 2002) have found that IGF, platelet-derived growth factor, and transforming growth factor $\beta$ have no growth-promoting activity in 3T3-L1 cells. However, Lee and Hossner observed that ultrafiltered, defatted $\mathrm{BC}$ stimulated adipogenesis in a manner independent of IGF and other growth factors (Lee and Hossner, 2002). The authors believe that BC contains unique growth-promoting peptides that are yet to be identified (Lyle et al., 1998). In our study, whole BC was also more effective in suppression of Fabp 4 activation, a binding protein that leads to activation of PPAR $\gamma$ and, consequently, adipogenesis.

\section{CONCLUSIONS}

Reporter assays provide a valuable high-throughput method of assessment of colostrum differences in whole and whey preparations. These assays could have other applications, for example they could be used as a cost-effective tool to study the role of different BC components in key biological pathways to determine their beneficial or deleterious properties. Antioxidant capacity of whole and whey BC correlate with their anti-inflammatory effects in cells. Whole BC exhibits higher anti-inflammatory, anti-adipogenic, and antioxidative effects on cells compared with whey BC.

\section{ACKNOWLEDGMENTS}

The authors are indebted to Fundação de Amparo à Pesquisa do Estado de São Paulo (FAPESP, \#2014/14937-7 and \#2017/02915-7, Brazil). The project was supported by National Institutes of Health (NIH) grants R21OD017244, the National Center for Research Resources UL1RR025755, UL1TR001070, and NCIP30CA16058 (OSUCCC), and the NIH Roadmap for Medical Research. The content is solely the responsibility of the authors and does not necessarily represent the official views of the National Center for Research Resources or the National Institutes of Health. This research was supported by the Parker Endowment at Ohio State University (no. 00100), the SEED Grant, and T. Kline Hamilton research award from College of Education and Human Ecology, Accelerator Grant from Office for Technology and Commercialization, and Brain Injury program from the Ohio State University. The content is solely the responsibility of the authors and does not necessarily represent the official views of the National Center for Advancing Translational Sciences (Bethesda, MD).

\section{REFERENCES}

Ahmadi, M., A.-B. Velciov, M. Scurtu, T. Ahmadi, and L. Olariu. 2011. Benefits of bovine colostrum in nutraceutical products J Agroalimentary Processes Technol. 17:42-45.

Albera, E., and M. Kankofer. 2009. Antioxidants in colostrum and milk of sows and cows. Reprod. Domest. Anim. 44:606-611.

An, M. J., J. H. Cheon, S. W. Kim, J. J. Park, C. M. Moon, S. Y. Han, E. S. Kim, T. I. Kim, and W. H. Kim. 2009. Bovine colostrum inhibits nuclear factor kappaB-mediated proinflammatory cytokine expression in intestinal epithelial cells. Nutr. Res. 29:275-280.

Appukutty, M., A. K. Radhakrishnan, K. Ramasamy, R. Ramasamy, A. B. Abdul Majeed, M. I. Noor, N. S. Safii, P. B. Koon, K. Chinna, and N. Haleagrahara. 2012. Colostrum supplementation protects against exercise-induced oxidative stress in skeletal muscle in mice. BMC Res. Notes 5:649.

Bachmeier, M., and G. Loffler. 1995. Influence of growth factors on growth and differentiation of 3T3-L1 preadipocytes in serum-free conditions. Eur. J. Cell Biol. 68:323-329.

Baker, R. G., M. S. Hayden, and S. Ghosh. 2011. NF-kappaB, inflammation, and metabolic disease. Cell Metab. 13:11-22. 
Belford, D. A., M. L. Rogers, G. L. Francis, C. Payne, F. J. Ballard, and C. Goddard. 1997. Platelet-derived growth factor, insulin-like growth factors, fibroblast growth factors and transforming growth factor beta do not account for the cell growth activity present in bovine milk. J. Endocrinol. 154:45-55.

Blais, M., M. Fortier, Y. Pouliot, S. F. Gauthier, Y. Boutin, C. Asselin, and M. Lessard. 2015. Colostrum whey down-regulates the expression of early and late inflammatory response genes induced by Escherichia coli and Salmonella enterica Typhimurium components in intestinal epithelial cells. Br. J. Nutr. 113:200-211.

Boney, C. M., H. Sekimoto, P. A. Gruppuso, and A. R. Frackelton Jr. 2001. Src family tyrosine kinases participate in insulin-like growth factor I mitogenic signaling in 3T3-L1 cells. Cell Growth Differ. $12: 379-386$.

Boudry, C., and A. Thewis. 2009. Bovine colostrum as a natural growth factor for newly-weaned piglets: A review. Bull. Univ. Agric. Sci. Vet. Med. Cluj-Napoca Anim. Sci. Biotechnol. 66:53-60.

Cervato, G., R. Cazzola, and B. Cestaro. 1999. Studies on the antioxidant activity of milk caseins. Int. J. Food Sci. Nutr. 50:291-296.

Chen, F., M. Wang, J. P. O'Connor, M. He, T. Tripathi, and L. E. Harrison. 2003. Phosphorylation of PPARgamma via active ERK1/2 leads to its physical association with p65 and inhibition of NF-kappabeta. J. Cell. Biochem. 90:732-744.

Choi, H. S., Y. G. Ko, J. S. Lee, O. Y. Kwon, S. K. Kim, C. Cheong, K. H. Jang, and S. A. Kang. 2010. Neuroprotective effects of consuming bovine colostrum after focal brain ischemia/reperfusion injury in rat model. Nutr. Res. Pract. 4:196-202.

Garg, B. D., H. Balasubramanian, N. S. Kabra, and A. Bansal. 2018. Effect of oropharyngeal colostrum therapy in the prevention of necrotising enterocolitis among very low birthweight neonates: A meta-analysis of randomised controlled trials. J. Hum. Nutr. Diet. $31: 612-624$.

Janusz, M., and A. Zablocka. 2010. Colostral proline-rich polypeptides-immunoregulatory properties and prospects of therapeutic use in Alzheimer's disease. Curr. Alzheimer Res. 7:323-333.

Kim, S. E., I. G. Ko, M. S. Shin, C. J. Kim, Y. G. Ko, and H. Cho. 2012. Neuroprotective effects of bovine colostrum on intracerebral hemorrhage-induced apoptotic neuronal cell death in rats. Neural Regen. Res. 7:1715-1721.

Korhonen, H., and A. Pihlanto. 2007. Technological options for the production of health-promoting proteins and peptides derived from milk and colostrum. Curr. Pharm. Des. 13:829-843.

Kuhn, K. A., N. A. Manieri, T. C. Liu, and T. S. Stappenbeck. 2014. IL-6 stimulates intestinal epithelial proliferation and repair after injury. PLoS One 9:e114195.

Lee, S. H., and K. L. Hossner. 2002. Effects of bovine colostral ultrafiltrates on growth and differentiation of 3T3-L1 preadipocytes. Biotechnol. Appl. Biochem. 36:205-212.

Lyle, R. E., J. D. Corley, and R. E. McGehee Jr. 1998. Human milk and infant formula can induce in vitro adipocyte differentiation in murine 3T3-L1 preadipocytes. Pediatr. Res. 44:798-803.
Neelima, R. Sharma, Y. S. Rajput, and B. Mann. 2013. Chemical and functional properties of glycomacropeptide (GMP) and its role in the detection of cheese whey adulteration in milk: A review. Dairy Sci. Technol. 93:21-43.

Pandey, N. N., A. A. Dar, D. B. Mondal, and L. Nagaraja. 2011. Bovine colostrum: A veterinary nutraceutical. J. Vet. Med. Anim. Health 3:31-35.

Raimondo, R. F. S., J. S. P. Ferrao, S. I. Miyashiro, P. T. Ferreira, J. P. E. Saut, D. B. Birgel, and E. H. Birgel Junior. 2019. The dynamics of individual whey protein concentrations in cows' mammary secretions during the colostral and early lactation periods. J. Dairy Res. 86:88-93.

Rosen, E. D., and B. M. Spiegelman. 2001. PPARgamma: A nuclear regulator of metabolism, differentiation, and cell growth. J. Biol. Chem. 276:37731-37734.

Scheller, J., A. Chalaris, D. Schmidt-Arras, and S. Rose-John. 2011. The pro- and anti-inflammatory properties of the cytokine interleukin-6. Biochim. Biophys. Acta 1813:878-888.

Shen, Q., C. Chitchumroonchokchai, J. L. Thomas, L. V. Gushchina, D. Disilvestro, M. L. Failla, and O. Ziouzenkova. 2014. Adipocyte reporter assays: Application for identification of anti-inflammatory and antioxidant properties of mangosteen xanthones. Mol. Nutr. Food Res. 58:239-247.

Shen, Q., K. M. Riedl, R. M. Cole, C. Lehman, L. Xu, H. Alder, M. A. Belury, S. J. Schwartz, and O. Ziouzenkova. 2015. Egg yolks inhibit activation of NF-kappaB and expression of its target genes in adipocytes after partial delipidation. J. Agric. Food Chem. 63:2013-2025.

Shing, Y., and M. Klagsbrun. 1987. Purification and characterization of a bovine colostrum-derived growth factor. Mol. Endocrinol. $1: 335-338$.

Stewart, M. G. 2008. Colostrinin: A naturally occurring compound derived from mammalian colostrum with efficacy in treatment of neurodegenerative diseases, including Alzheimer's. Expert Opin. Pharmacother. 9:2553-2559.

Tontonoz, P., and B. M. Spiegelman. 2008. Fat and beyond: The diverse biology of PPARgamma. Annu. Rev. Biochem. 77:289-312.

Wardyn, J. D., A. H. Ponsford, and C. M. Sanderson. 2015. Dissecting molecular cross-talk between Nrf2 and NF-kappaB response pathways. Biochem. Soc. Trans. 43:621-626.

Yadav, R., T. Angolkar, G. Kaur, and H. S. Buttar. 2016. Antibacterial and antiinflammatory properties of bovine colostrum. Recent Pat. Inflamm. Allergy Drug Discov. 10:49-53.

Yamashita, A. S., T. Belchior, F. S. Lira, N. C. Bishop, B. Wessner, J. C. Rosa, and W. T. Festuccia. 2018. Regulation of metabolic disease-associated inflammation by nutrient sensors. Mediators Inflamm. 2018:8261432.

Zanardo, V., R. Golin, M. Amato, D. Trevisanuto, F. Favaro, D. Faggian, and M. Plebani. 2007. Cytokines in human colostrum and neonatal jaundice. Pediatr. Res. 62:191-194. 